\title{
Effect of Green Tea Amino Acid L-Theanine on Physiological Responses: A Protocol for Clinical Trial
}

\author{
Jackson Williams ${ }^{1}$, Nathan M. D’Cunha ${ }^{1}$, Nicola Anstice ${ }^{1}$, \\ Andrew McKune ${ }^{1,2,3}$ and Nenad Naumovski ${ }^{*}$ \\ ${ }^{1}$ Faculty of Health, University of Canberra, Canberra, ACT, 2601, Australia; ${ }^{2}$ Discipline of Biokinetics, Exercise and Leisure \\ Sciences, School of Health Sciences, University of KwaZulu-Natal, Durban, KwaZulu-Natal, South Africa; \\ ${ }^{3}$ Research Institute of Sport and Exercise, University of Canberra, Canberra, ACT, 2605, Australia
}

\begin{abstract}
Background and objectives: L-theanine (L-THE) is a green tea-derived amino acid, consumed for its many benefits, including improved cardiovascular health, anxiolytic effects, antioxidant properties, and its effect on instigating a state of relaxed alertness. The aim of this clinical trial is to evaluate the effectiveness of the amino acid L-THE embedded in functional food whey protein mango sorbet, its related stress effects on physiological responses, state of alertness, and focus, and the accuracy of eye movements post-consumption.
\end{abstract}

Methods: Twenty-one healthy males, aged $18-65$, will be recruited for this study. Participants will be required to consume a mango sorbet (without L-THE) and mango sorbet containing $0.2 \mathrm{~g}$ of L-THE and a placebo or $0.2 \mathrm{~g}$ pure L-THE within a capsule, after an overnight fast. L-THE exerts its effects 30-50 minutes post consumption, lasting up to 90 minutes. Participants will perform a series of visual functional tests, including habitual visual acuity, contrast sensitivity and measurements of saccadic eye movements after the consumption of the food products at 15-minute intervals to measure their state of alertness and fatigue. Salivary cortisol will be measured every 30 minutes; blood pressure, heart rate and heart rate variability responses will also be measured every 10 minutes.

Discussion: The use of L-THE as a functional additive may provide potential therapeutic stress benefits when consumed alongside food products. The results of this protocol study will ultimately determine whether L-THE embedded within mango sorbet at physiologically relevant levels can alter the stress response and exhibit its effect on eye fatigue and concentration.

\section{Introduction}

Functional foods describe designed food items that have been shown to be health-enhancing; however, they contain bioactive non-'naturally occurring' compounds, with the aim of increasing

Keywords: L-theanine; Bioactives; Clinical trial protocol; Physiological responses. Abbreviations: ANS, autonomic nervous system; BP, blood pressure; CS, contrast sensitivity; HF, high frequency; HPA, hypothalamic-pituitary-adrenal; HR, heart rate; HRV, heart rate variability; LF, low frequency; L-THE, L-theanine; MAP, mean arterial pressure; PNS, parasympathetic nervous systems; SEMs, saccadic eye movements; SNS, sympathetic nervous system; SPIRIT, Standard Protocol Items: Recommendations for Interventional Trials; VA, visual acuity.

Received: July 02, 2020; Revised: July 28, 2020; Accepted: August 03, 2020

*Correspondence to: Nenad Naumovski, Faculty of Health, University of Canberra, P.O. Box 5018, Bruce ACT, 2617, Australia. Tel: +61-(0)2-6206-8719; E-mail: nenad. naumovski@canberra.edu.au

How to cite this article: Williams J, D'Cunha NM, Anstice N, McKune A, Naumovski N. Effect of Green Tea Amino Acid L-Theanine on Physiological Responses: A Protocol for Clinical Trial. Exploratory Research and Hypothesis in Medicine 2020;000(000):000-000. doi: 10.14218/ERHM.2020.00048 product functionality. ${ }^{1}$ This relatively new concept of health-promoting functional foods is a significant part of a growing global food trend that is becoming a secondary component of medical treatment. ${ }^{1}$ Functional foods provide a potential opportunity to rely less on the use of pharmaceuticals, resulting in high demand for beverages and food products that include health-promoting active ingredients. ${ }^{2}$

The potential functional food additive L-Theanine (L-THE) is a green tea amino acid that has shown to increase relaxation, ${ }^{3}$ decrease sleep disturbances ${ }^{4}$ and decrease anxiety and the stress response $\mathrm{s}^{5}$ in its pure form in humans (healthy and non-healthy) via capsule intake. The recent increase in the commercial availability of this amino acid in its pure form has sparked an increase in its use globally. Furthermore, several commercially available food products contain L-THE, such as chewing gum, drinks and chocolate, each with their own respective claims related to health benefits. ${ }^{6,7}$ However, there is a paucity of clinically relevant evidence to support claims on the functionality embedded within food, as a functional food product. The processes utilized in the preparation of functional food products (i.e. high temperatures and changes in 
$\mathrm{pH})$ can reduce the functionality of L-THE, while its combination with some ingredients (such as caffeine and other amino acids) may interfere with its absorption. ${ }^{3}$

Various studies report stress lowering effects (post-200 mg consumption in its pure form), demonstrated physiologically as reductions in heart rate $(\mathrm{HR})^{8}$ as well as reductions in salivary cortisol that indicate the potential interaction between L-THE and hypothalamic-pituitary-adrenal (HPA) axis system responses. ${ }^{8,9}$ Moreover, consumption of L-THE $(250 \mathrm{mg})$ is reported to halt the increase in blood pressure (BP) ${ }^{10}$ that is thought to occur due to L-THE's effect on blood vessels in the peripheral nervous system via the mechanism of vasodilation, which could highlight the effect of L-THE on cardiovascular structures and the interaction between vascular tone and the BP response. ${ }^{3}$ These stress-related responses are heavily regulated by the autonomic nervous system (ANS), comprising the parasympathetic nervous systems (PNS) and sympathetic nervous system (SNS) that are key to maintaining allostasis, the process of achieving homeostatic stability through change. ${ }^{11,12}$

It is postulated that individuals with high allostatic resilience display healthier ANS responses. HR variability (HRV) is considered a reliable marker for ANS responses caused by internal and external stressors. It is specifically measured by changes between the highest points ( $\mathrm{R}-\mathrm{R}$ interval) of the heartbeat intervals observed on an electrocardiogram (QRS complex). ${ }^{1}$ Due to its effect on the stress response, we hypothesize that L-THE will influence the physiological outcomes of the ANS, including but not limited to HR, HRV, BP and the HPA stress response via the salivary biomarker cortisol. ${ }^{3,5,8}$ The consumption of L-THE has also been reported to have a profound effect on concentration and increasing alertness, which may improve the speed and accuracy of eye movements, and due to its effects on glutamatergic neurotransmission, may also alter neurophysiological responses in the retina. ${ }^{13}$ Furthermore, L-THE is proposed to affect cognitive performance and increase alertness ${ }^{14}$; therefore, investigating saccadic eye movements (SEMs; the shift in rapid eye movements between objects of visual interest), as well as contrast sensitivity (CS) and visual acuity (VA), which are markers of retinal function, may further the understanding of neurophysiological responses in humans post-L-THE consumption. Despite the gap in the literature that establishes a relationship between everyday food consumption and L-THE, investigating these responses may potentially highlight a relationship between an L-THE-containing functional food and the stress response.

Therefore, the aim of this clinical trial is to evaluate the effectiveness of the amino acid L-THE and its related health effects on physiological and visual responses as a functional food ingredient in a whey protein mango sorbet. The primary objective of this clinical trial is to determine the physiological responses of L-THE $(200 \mathrm{mg}$ ) imbedded in the food matrix (mango sorbet) in comparison to supplementation of pure L-THE, implemented in a randomized, double-blind, placebo-controlled crossover design. The results of this study will form the basis for a potential new commercial food product that can provide an alternate new delivery method of L-THE at physiologically relevant levels to increase relaxation and alertness and reduce stress.

\section{Methods}

This protocol paper has been completed in accordance with the Standard Protocol Items: Recommendations for Interventional Trials (SPIRIT) checklist. This project has been approved by the
Human Research Ethics Committee of the University of Canberra (2019-1534) and is registered by the Australian New Zealand Clinical Trials Registry: 12619000815167, Universal Trial Number: U1111-1228-6809. Participants will be informed about the study protocols and provided written consent. On completion of written consent, participants will then be deemed eligible to be screened to determine eligibility for participation. Recruitment of participants will occur at the University of Canberra Hospital: Specialist Centre for Rehabilitation, Recovery and Research, ACT Australia. In addition, clinical testing and all data collection will be conducted at the University of Canberra Health Hub, Australia in a standard optometry examination room in the University of Canberra Eye Clinic.

We will include healthy males, aged 18-65 years-old. Females will not be included in this study due to the potential interferences associated with menstrual hormonal changes on the stress responses that will be measured in this study. ${ }^{15}$ We will exclude participants if they consume functional foods that include stanol or sterol ester-containing margarine, or commercial dietary products/supplements associated with weight loss. Participants will be excluded if they currently experience or have had any known active pulmonary, hematologic, hepatic, gastrointestinal, renal, premalignant, malignant illnesses, or have diabetes (type I and type II) or any thyroid dysfunction, following previously conducted research in similar supplements. ${ }^{16}$ Participants will also be excluded if they have any of the following visual impairments: glaucoma, cataracts, age-related macular degeneration, strabismus, amblyopia, traumatic brain injury, blindness/partial sight or any other retinal disorder that may affect an individual's visual function. For the purposes of the physiological measurements in this study, a qualified optometrist will oversee the interventions related to eyesight.

\section{Interventions}

The mango sorbet used in this study was developed and modified based on the formulation found in a recently published manuscript. ${ }^{16}$ The L-THE (Suntheanine ${ }^{\mathrm{TM}}$; Taiyo Kagaku Co., Ltd, Mie, Japan) was purchased from Ingredient Resources Australia and New Zealand Pty/Ltd (Sydney, NSW, Australia) and was the functional additive $(200 \mathrm{mg} / 100 \mathrm{~g} \mathrm{w} / \mathrm{w})$. The mangos, erythritol and whey protein concentrate were purchased from commercial suppliers. Despite L-THE being consumed 'normally' as a hot beverage, as a delivery matrix, sorbet was selected due to the favorable storage conditions of L-THE, such as low temperature (less than $4^{\circ} \mathrm{C}$ ) and slightly acidic environment ( $\mathrm{pH}$ range 5-6). ${ }^{17}$

The levels of L-THE administered in previous clinical trials range from a bolus of $100-900 \mathrm{mg}$ daily, which is equivalent to the L-THE 'normal' concentration found in daily consumption of $6-12$ cups of green tea, ${ }^{14,18,19}$ and the dosage of L-THE (200 mg) was selected based on the findings of previous studies that demonstrated the physiological effects of L-THE consumption related to the stress response and concentration. . $^{3,20,21}$ Whey protein was selected due to its ability to provide structural integrity to the sorbet. The development of the mango sorbet will adhere to hazard analysis and critical control points principles and good manufacturing practices. ${ }^{22}$ The mango sorbet will be prepared and adapted based on methods described in previous studies by Naumovski et $a l .{ }^{16}$ For the preparation of the encapsulated interventions, gelatin capsules (size 3) will be filled with microcrystalline cellulose filler acting as the placebo, whilst $200 \mathrm{mg}$ L-THE will be used to fill the active treatment capsules. 


\section{Intervention toxicology}

Relatively recent health concerns about the use of green tea extracts further support the appropriate use of L-THE in its pure form rather than alongside other compounds that are commonly found in green tea and its extracts. Green tea extract-based products and their consumption in combination with protein supplements have safety risks; in particular, overconsumption of the green tea extract Garcinia cambogia has been reported to cause pattern liver injury. ${ }^{23}$ However, despite their mildly beneficial effects, when consumed in excessive doses, these green tea extracts' compounds have the potential to cause significant health problems. ${ }^{23}$ To our knowledge, there are no adverse effects related to excessive intake of L-THE and Food Standards Australia and New Zealand recommend L-THE as a substance that can be added to herbal infusion tea bag/sachet (i.e. powder) at up to $2 \mathrm{~g} / 100 \mathrm{~g}$ of total natural and added substance. ${ }^{24}$

\section{Clinical sequence}

The primary outcomes considered for this trial will be the change in biomarkers at pre-determined time points between 0 to 90 minutes. These measurements and their respective time points include:

\section{Physiological responses}

The effects of L-THE on sympathetic nervous system regulation of BP is of interest. The use of L-THE in its pure form indicate stress lowering effects and relaxation, expressed physiologically in the form of reductions in BP in response to a high stressors in adults. ${ }^{25}$ Furthermore, consumption of L-THE $(250 \mathrm{mg})$ is reported to ameliorate the increase in BP related to certain stressful stimuli. ${ }^{10}$ This provides a rationale to assess its effect on the peripheral nervous system and cardiovascular structures that may affect vascular tone and the BP response. ${ }^{3}$

a. Changes in systolic BP (SBP)

b. Changes in diastolic BP (DBP)

c. Changes in mean arterial pressure (MAP) as determined by the formula:

$$
D B P+\frac{S B P-D B P}{3}
$$

All BP will be measured at 10-minute intervals, up to 90 minutes.

Changes in HRV will reflect the effects of L-THE on cardiac autonomic nervous system regulation, in particular relating to PNS activity (as indicated by changes in high frequency (HF) and low frequency (LF)). ${ }^{1}$ This interaction between L-THE and HRV may potentially highlight the response to food consumption and its relationship with disease. ${ }^{26}$

d. HR and HRV

i. Changes in HR as the total number of contractions/ beats per minute

ii. Changes in HRV as determined by the HF band (range $0.15-0.4 \mathrm{~Hz}$ ) that reflects parasympathetic nervous system (PNS) activity ${ }^{26}$

iii. Changes in HRV as determined by LF band (range $0.04-0.15 \mathrm{~Hz}$ ) that represents baroreflex function as well as PNS activity ${ }^{27}$

The HR and HRV will be measured continuously for up to 90 minutes.

VA and contrast sensitivity (CS) are used as biomarkers of gross retinal function and are of interest as previous research has found L-THE alters neurophysiological responses in the retina. ${ }^{13}$ Analysis of SEMs provides an indication of cognitive processing and thus testing a visual reaction task, such as SEMs, could be a strong indicator of an individual's state of alertness, focus, and eye movement control. ${ }^{28,29}$ Further, L-THE is known to strongly influence cognitive performance as well as increasing alertness ${ }^{14}$ and investigating SEMs may further the understanding of the neurophysiological responses to L-THE in humans.

e. Changes in habitual VA; the ability to high spatial frequency details of shapes or objects at a given distance

f. Changes in CS; sensitivity of the visual system to detect differences in the brightest and dimmest parts of a target

g. Changes in SEMs; rapid fixational eye movements designed to shift both eyes in the direction of an object of visual interest

The following changes will be measured:

i. Total number of fixations

ii. Mean number of fixations in a row

iii. Total number of rightwards saccades

iv. Total number of regressions

v. Mean number of regressions in a row

vi. Total number of regressions/total number of fixations

The VA, CS and SEMs will be measured at 15-minute intervals, up to 90 minutes.

\section{Biochemical responses}

Cortisol is secreted in a pulsatile manner that is reflective of the body's natural circadian pattern and is reflective of an individual's allostatic load that adapts in response to environmental changes and experiences, such as the consumption of food. ${ }^{8,30}$ It is also secreted in response to adverse aversive events of acute stress as well as to metabolism fluctuations. ${ }^{31,32}$ It has been postulated that doses of L-THE show positive interactions that result in the reduction of salivary cortisol levels. ${ }^{6,33}$

a. Changes in salivary cortisol

Salivary cortisol will be measured at 30-minute intervals, up to 90 minutes in duration.

\section{Participant timeline}

Participants will be required to attend a total of six visits: one information visit that will last up to 15 minutes and five clinical testing visits (each lasting up to 120 minutes). For the purposes of the five clinical testing visits, participants will receive no intervention in the first clinic visit, in order to establish baseline measurements, whilst the remaining four visits will be randomized for the consumption of one product per clinic (Fig. 1). These food products will include the two treatment products, both containing $200 \mathrm{mg}$ of L-THE, one in the form of a whey protein mango sorbet, whereas the latter will be embedded within a gelatin capsule. The remaining two (placebo) interventions include a whey protein mango sorbet and the second is encapsulated microcrystalline cellulose, both without the added L-THE. Each clinic visit must be separated by a minimum of 48 hours washout period, to allow the metabolism and clearance of L-THE from circulation. ${ }^{21}$

Prior to attending clinic visits two through six, participants will be required to fast overnight (or for at least 8 hours prior to the 


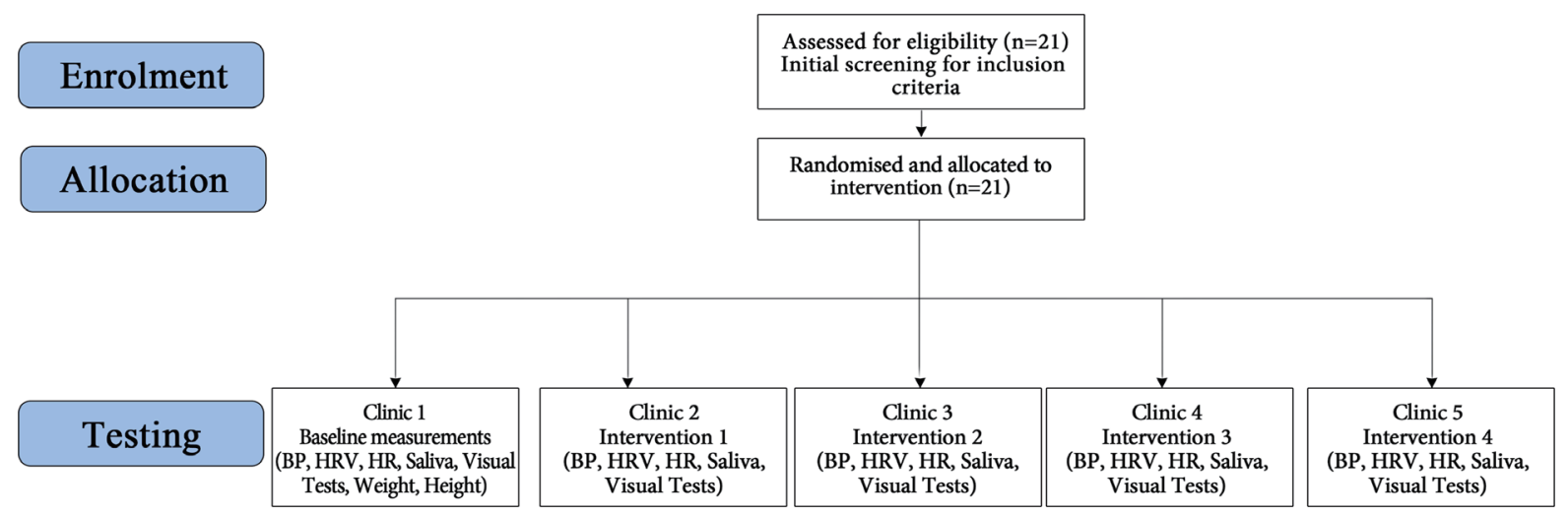

Fig. 1. Participant timeline/schedule of events.

visit) except for water consumption. Participants will be required to refrain from alcohol for 24 hours and caffeine for 12 hours prior to the commencement of the clinic visit, in order to prevent the potential for interaction between other bioactive compounds and the currently developed mango sorbet food matrix.

All physiological measurements for all clinic visits will begin after 10 minutes of each participant being seated in a sedentary position to ensure minimal fluctuation of BP or HR responses. Data accumulation will begin after complete consumption of the respective food product or capsule intervention (Table 1).

\section{Sample size, allocation and concealment}

The sample size calculations are based on detecting changes in SEM responses. The total number of participants needed for this trial to ensure statistical significance $($ power $=0.8$, alpha $=0.05$ ) is 21 (obtained using the Harvard sample size calculator (hedwig. mgh.harvard.edu/sample_size/js/) as well as methods from previous studies. ${ }^{3,6}$ Based on a standard $80 \%$ power, alpha was set to 0.05 and a standard deviation of $55 \mathrm{~ms}$ in the latency of SEM, with a minimal detectable difference between mean SEM latency of 50 $\mathrm{ms}$. A total of $50 \times 100 \mathrm{~g}$ sorbets $(25$ with the added $200 \mathrm{mg}$ L-THE and 25 without the added L-THE) as well as $50 \times$ sets of capsules ( 25 with the $200 \mathrm{mg} \mathrm{L-THE}$ and 25 with microcrystalline cellulose filler) will be produced, accounting for the potential withdrawal of participants from the trial. Participants will be recruited following responses to an advertising poster placed on the notice boards at the University of Canberra (Canberra, ACT, Australia) as well as email outlets delivered to all staff and students.

The products (mango sorbets and capsules) will be assigned selected numbers using the random number generation function within the Microsoft Excel 2016 (v16, Microsoft, Pennant Hills, NSW, Australia), before randomly assigning each category of the treatment to participants for consumption using the online application Research Randomiser (https://www.randomiser.org). Allocated randomized treatment sequences will be achieved using a block randomization protocol (block size of 4) with 25 sets of 4 random sequenced numbers per intervention (randomizer.org) to ensure the mango sorbet and mango sorbets with the added LTHE administration are balanced between the participants. The sequence code will be placed in a sealed envelope and stored in a lockable cabinet belonging to a researcher not involved with the study. One of the investigators (JW) will solely be responsible for the enrolment of participants, assigning each individual a unique participant number, and responsible for providing each participant with their respective coded food product or capsule intervention. The code will be revealed only at the end of the trial once all participants have finalized all of their visits. Participant privacy and confidentiality will be assured at all times. All data (medical information, physiological results, and visual results) collected will be de-identified to protect participant anonymity. All academic journal publications that result from this clinical trial will be reported using de-identified data so that no individual data (personal details such as name, age and height) will be included. All collected biomarker data will be re-identifiable using a coding system allocated to each participant that allows for blinded data analysis. All participant information and data collected will be stored securely on a password-protected computer throughout the project and then stored at the University of Canberra for the required 15-year period after which it will be destroyed according to University of Canberra protocols. Any participant that voluntarily discontinues or deviates from the study protocol will have their personal information and data destroyed as per University of Canberra protocols.

\section{Data collection methods}

Changes in BP will be determined and adapted following the guidelines for the $2^{\text {nd }}$ Australian National BP study. ${ }^{34}$ BP via arm cuff will be determined using the Visage Digital BP Monitor ABO 523 (Emergo, Sydney, Australia) calibrated against an OMRON HEM7130 BP monitor (Kyoto, Japan). Continuous readings will be taken on participants' left arms for up to 90 minutes at each 10-minute time interval. ${ }^{16}$

HRV will be measured via an HR belt (Scosche Rhythm+ Armband HR Monitor; Oxnard, CA, USA) that measures HR and the time between R-R intervals of successive heartbeat complexes using Kubios HRV Standard 3.0.2 diagnostic device software (Kuopio, Finland). Participants are required to wear the belt placed over the intermediate cephalic vein on the upper left forearm whilst seated in a sedentary position during the clinic visit. ${ }^{35}$

Participants will have their threshold habitual VA measured seven times using a computer-generated Early Treatment of Diabetic Retinopathy Study chart during each of the respective clinic visits, occurring at 15-minute intervals from the commencement of the clinic for up to 90 minutes. VA will be measured using appropriately-scaled Sloane letters on the Thomson Software Solutions system (Hatfield, UK) located, via a mirror, at a viewing distance of $7.1 \mathrm{~m}$. Letters on each line will be randomized using the Thomson testing software's randomization function at each 15-minute interval to prevent learning effects. Whilst covering one eye with 


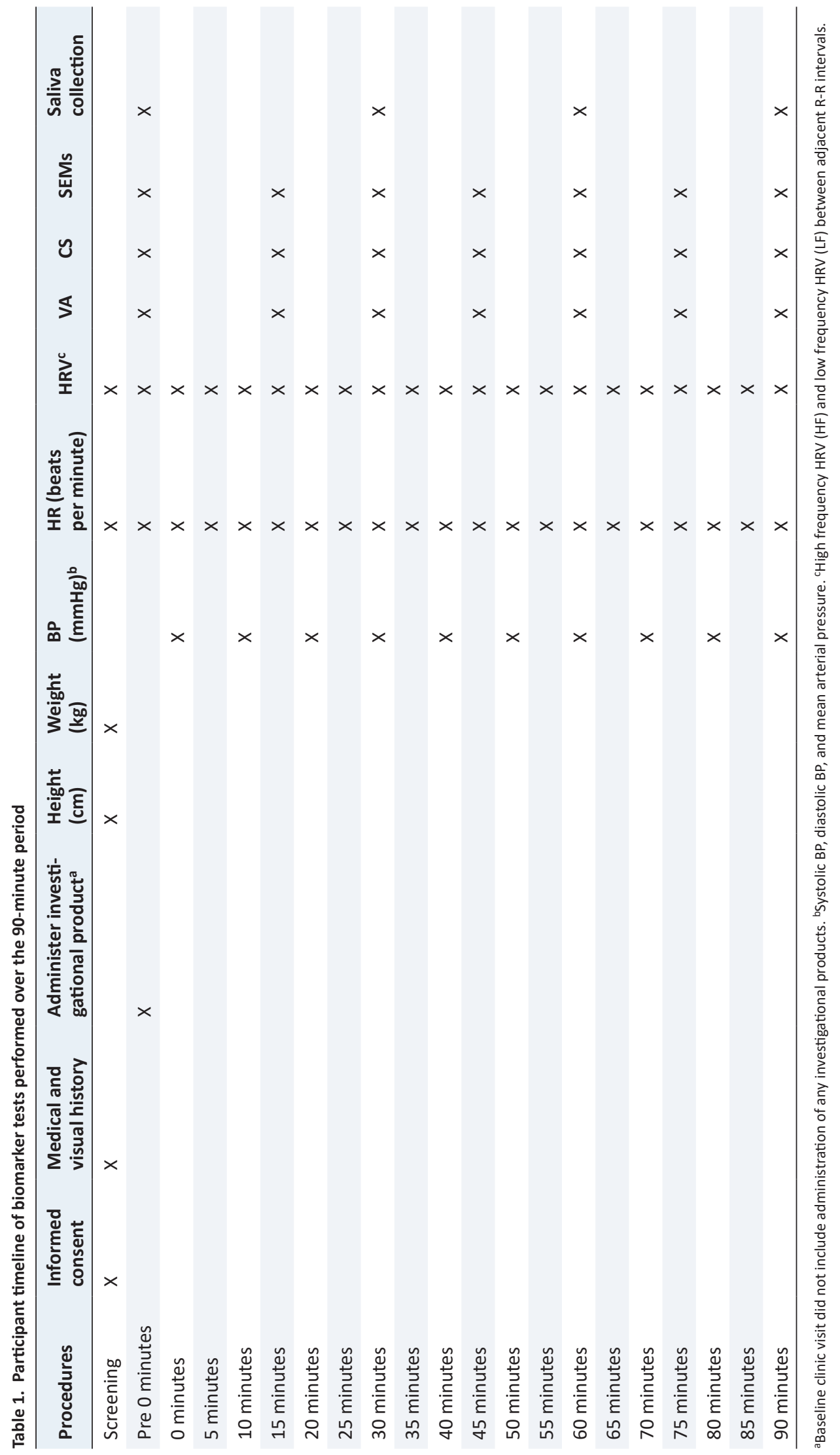


an occluder, and with the participants' habitual correction (current glasses, contact lenses, or unaided) in place, participants will be instructed to read each line of five letters. Starting with the largest letters, participants will be prompted to identify each letter from left to right and continue down the chart until the participant can no longer correctly identify four of the five letters on a single row. This process will be repeated with the other eye. VA will be scored using a logarithmic minimum angle of resolution notation, on an optotype-by-optotype scoring basis, giving credit for all correctly identified letters.

To measure CS, participants will be required to complete seven CS tasks during each of the respective clinics, occurring at each 15-minute interval from the commencement of the clinic visit, up to 90 minutes. The CS task will be measured using a computergenerated CS test (Thomson Software Solutions) with a Sloan letter set (fixed size equivalent to 6/24) and based on the principles of the Pelli-Robson CS test. ${ }^{36}$ Letters will be randomized using the Thomson testing software's randomization function at each 15-minute interval to prevent learning effects. Measurements will be conducted monocularly with the participant's habitual correction in place. Participants will be instructed to read each triplet of letters of equal contrast aloud from a viewing distance of 1 meter from a 24-inch monitor. The letter contrast will then be reduced in $0.15 \log$ CS steps until the participant cannot correctly identify any two of the three letters in the triplet. This process will be repeated with the other eye.

For SEMs, the TobiieyeX 4C eye tracking device (Stockholm, Sweden) will be used to record SEMs using the Thomson Software Solutions Clinical Eye Tracker software version 18.04. Participants will be required to complete seven 1-minute SEMs tasks during each of the respective clinic visits, occurring at each 15-minute interval from the commencement of the clinic visit, up to 90 minutes. Participants will be required to be in a sedentary position with a laptop screen at eye height no more than $75 \mathrm{~cm}$ from their eyes and prompted to accurately focus on two red stimulus dots that constantly interchange on a black background (stimulus size: 10; amplitude of SEMs \pm 20 degrees; angle between two fixation points: 0 degrees (horizontal presentation of stimuli); timing of alternating stimuli: $400 \mathrm{~ms}$ ).

To measure salivary cortisol, the collection schedule will require participants to provide four 2-mL saliva samples for the baseline clinic visit (the first day with no intervention) and for each of the respective clinic visits (one saliva sample prior to the food/capsule consumption and three post-food consumption, at time intervals of 30,60 and 90 minutes). The collection times are intended to reflect the activity in which L-THE reaches peak blood circulation post-pure $200 \mathrm{mg}$ intake. ${ }^{5}$ Salivary cortisol levels peak approximately 20 minutes post-HPA axis activation; therefore, if stress is increased during the clinic visit, this will reflect the relationship between L-THE and stress. Participants will be instructed with a demonstration on how to provide saliva samples via the passive drool method following protocols described by McKune et al. ${ }^{30}$ (2014) that is to be collected over a 3-minute unstimulated period, wherein participants lean forward and passively let saliva fall into a collection tubule. ${ }^{30,37}$ Saliva samples will be stored in a freezer $\left(-18{ }^{\circ} \mathrm{C}\right)$ until cortisol analysis. ${ }^{37}$ The time of collection is to be recorded for all samples. Salivary cortisol is to be measured by enzyme-linked immunosorbent assay kits purchased from a commercial supplier (Stratech Scientific APAC Pty, Ltd, Mona Vale, NSW, Australia) and analyzed in duplicate, based on the manufacturer's instructions (Salimetrics, LLC, State College, PA, USA). Samples from the same participant will be tested using the same analysis kit to avoid between-person variability.

The obtained HR and HRV R-R interval data will be exported from the HR monitors via the Elite HRV phone application (Elite HRV Inc, Asheville, NC, USA) for time domain and spectral HRV analysis using the Kubios HRV Standard 3.0.2 diagnostic device software and exported as a note file. All R-R interval artefacts will be manually corrected using previously described methods ${ }^{36}$ and the HF and LF HRV values will be log-transformed prior to analysis. Total area under the curve, the primary model for analysis for time effects for between-group time relationships for the entire 0-90 min block for salivary cortisol, BP, visual and all HR data will be analyzed using the repeated measures ANOVA. If data is not normally distributed, then the non-parametric equivalent will be applied. Level of significance will be set at alpha of 0.05 . Data will be analyzed using the SPSS v25 (IBM Corp, Armonk, NY, USA).

In the event of a clinical trial adverse event (i.e. any untoward medical occurrence in a subject due to administered food product or external factor, without regard to the possibility of a causal relationship), the subject will cease taking the food product immediately and be withdrawn from the study. Further, the lead researchers will be responsible for ensuring the participant receives the relevant medical attention.

\section{Discussion}

These stress responses in humans are strongly regulated by the ANS, which plays a significant role in maintaining a state of homeostasis. ${ }^{11,12}$ There is the potential for consumption of food products containing the amino acid L-THE to affect the physiological stress responses determined by vital signs HR, HRV, and BP. Furthermore, due to the already established link between previous studies involving L-THE and concentration, the results of this study will potentially elucidate the link between L-THE consumption, fatigue and concentration as measured visual biomarkers. The processes involved in this will potentially demonstrate these relationships by embedding L-THE within a whey protein-based mango sorbet and its effects on the physiological and visual responses in the body post-consumption acutely, up to 90 minutes. The measurements will further aid researchers to understand the activities of L-THE integrated into food matrixes comparatively against L-THE in its pure form, which may improve nutritional guidelines to improve overall health. The potential for L-THE to elicit such a response would provide added value to the scientific community and is one method that can potentially further establish the link between LTHE consumed as a functional food additive and form the basis for a potential new commercial food product that can provide an alternate new delivery method to exert its biological effects in the human population.

\section{Acknowledgments}

This research was supported by the Faculty of Health Research Support Funding, University of Canberra, Canberra, ACT, Australia.

\section{Funding}

J.W. is supported by an Australian Government Research Training Program Scholarship. N.M.D is supported by a Dementia Australia Research Foundation PhD Scholarship. For this project, N.N. has received the Faculty of Health Research Support Funding from the 
University of Canberra (Canberra, ACT, Australia). All other authors have no sources of funding to declare.

\section{Conflict of interest}

The researchers of this project and the authors declare no conflicts of interest.

\section{Author contributions}

Conceptualization and study design (JW, NA, AM and NN), collection management (JW), formal analysis and data interpretation (JW, ND, NA, AM and NN), methodology (JW, NA, AM and NN), project administration $(\mathrm{NN})$, supervision (NA, AM and $\mathrm{NN}$ ), visualization (JW and ND), writing, including review and editing (JW, $\mathrm{ND}, \mathrm{NA}, \mathrm{AM}$ and $\mathrm{NN})$.

\section{References}

[1] Williams J, McKune AJ, Georgousopoulou EN, Kellett J, D'Cunha NM, Sergi $D$, et al. The Effect of L-Theanine Incorporated in a Functional Food Product (Mango Sorbet) on Physiological Responses in Healthy Males: A Pilot Randomised Controlled Trial. Foods 2020;9(3):371. doi:10.3390/foods9030371.

[2] Harrison L, Smith R. Developing food products for consumers concerned with physical activity, sports, and fitness. Osborn S, Morley W. (eds) Developing Food Products for Consumers with Specific Dietary Needs. Woodhead Publishing. 2016:215-239. doi:10.1016/B978-008-100329-9.00011-6.

[3] Juneja LR, Chu DC, Okubo T, Nagato Y, Yokogoshi H. L-theanine-a unique amino acid of green tea and its relaxation effect in humans. Trends Food Sci Technol 1999;10(6-7):199-204. doi:10.1016/S09242244(99)00044-8.

[4] Kim S, Jo K, Hong K-B, Han SH, Suh HJ. GABA and I-theanine mixture decreases sleep latency and improves NREM sleep. Pharm Biol 2019:57(1):65-73. doi:10.1080/13880209.2018.1557698.

[5] Williams JL, Everett JM, D'Cunha NM, Sergi D, Georgousopoulou EN, Keegan RJ, et al. The Effects of Green Tea Amino Acid L-Theanine Consumption on the Ability to Manage Stress and Anxiety Levels: a Systematic Review. Plant Foods Hum Nutr 2020;75(1):12-23. doi:10.1007/s11130-019-00771-5.

[6] White DJ, de Klerk S, Woods W, Gondalia S, Noonan C, Scholey AB. Anti-Stress, Behavioural and Magnetoencephalography Effects of an I-Theanine-Based Nutrient Drink: A Randomised, DoubleBlind, Placebo-Controlled, Crossover Trial. Nutrients 2016;8(1):53. doi:10.3390/nu8010053.

[7] Vuong QV, Bowyer MC, Roach PD. L-Theanine: properties, synthesis and isolation from tea. J Sci Food Agric 2011;91(11):1931-1939. doi:10.1002/jsfa.4373.

[8] Kimura K, Ozeki M, Juneja LR, Ohira H. L-Theanine reduces psychological and physiological stress responses. Biol Psychol 2007;74(1):3945. doi:10.1016/j.biopsycho.2006.06.006.

[9] McKune AJ, Bach CW, Semple SJ, Dyer BJ. Salivary cortisol and alphaamylase responses to repeated bouts of downhill running. Am J Hum Biol 2014;26(6):850-855. doi:10.1002/ajhb.22605.

[10] Rogers PJ, Smith JE, Heatherley SV, Pleydell-Pearce CW. Time for tea: mood, blood pressure and cognitive performance effects of caffeine and theanine administered alone and together. Psychopharmacology (Berl) 2008;195(4):569-577. doi:10.1007/s00213007-0938-1.

[11] Botek M, Krejčí J, De Smet S, Gaba A, McKune AJ. Heart rate variability and arterial oxygen saturation response during extreme normobaric hypoxia. Auton Neurosci 2015;190:40-45. doi:10.1016/j. autneu.2015.04.001.

[12] Speer KE, Semple S, Naumovski N, D'Cunha NM, McKune AJ. HPA axis function and diurnal cortisol in post-traumatic stress disorder: A systematic review. Neurobiol Stress 2019;11:100180. doi:10.1016/j. ynstr.2019.100180.

[13] Kahathuduwa CN, Dassanayake TL, Amarakoon AMT, Weerasinghe VS. Acute effects of theanine, caffeine and theanine-caffeine combination on attention. Nutr Neurosci 2017;20(6):369-377. doi:10.1080 /1028415x.2016.1144845.

[14] Camfield DA, Stough C, Farrimond J, Scholey AB. Acute effects of tea constituents L-theanine, caffeine, and epigallocatechin gallate on cognitive function and mood: a systematic review and meta-analysis. Nutr Rev 2014;72(8):507-522. doi:10.1111/nure.12120.

[15] Maki PM, Mordecai KL, Rubin LH, Sundermann E, Savarese A, Eatough $\mathrm{E}$, et al. Menstrual cycle effects on cortisol responsivity and emotional retrieval following a psychosocial stressor. Horm Behav 2015;74:201-208. doi:10.1016/j.yhbeh.2015.06.023.

[16] Naumovski N, Blades BL, Roach PD. Food Inhibits the Oral Bioavailability of the Major Green Tea Antioxidant Epigallocatechin Gallate in Humans. Antioxidants (Basel) 2015;4(2):373-393. doi:10.3390/ antiox4020373.

[17] PubChem: National Center for Biotechnology Information. L-Theanine. Available from: http://pubchem.ncbi.nlm.nih.gov/compound/LTheanine\#section=Top. Accessed July 25, 2020.

[18] Sarris J, Byrne GJ, Cribb L, Oliver G, Murphy J, Macdonald P, et al. Ltheanine in the adjunctive treatment of generalized anxiety disorder: A double-blind, randomised, placebo-controlled trial. J Psychiatr Res 2019;110:31-37. doi:10.1016/j.jpsychires.2018.12.014.

[19] Unno K, Tanida N, Ishii N, Yamamoto H, Iguchi K, Hoshino M, et al. Anti-stress effect of theanine on students during pharmacy practice: positive correlation among salivary alpha-amylase activity, trait anxiety and subjective stress. Pharmacol Biochem Behav 2013;111:128135. doi:10.1016/j.pbb.2013.09.004.

[20] Mason R. $200 \mathrm{mg}$ of Zen: L-Theanine Boosts Alpha Waves, Promotes Alert Relaxation. Altern Complement Ther 2001;7(2):91-95. doi:10.1089/10762800151125092.

[21] Scheid L, Ellinger S, Alteheld B, Herholz $\mathrm{H}$, Ellinger J, Henn T, et al. Kinetics of L-theanine uptake and metabolism in healthy participants are comparable after ingestion of L-theanine via capsules and green tea. J Nutr 2012;142(12):2091-2096. doi:10.3945/jn.112.166371.

[22] Australian Institute of Food Safety. The Seven Principles of Hazard Analysis and Critical Control Points 2013. Available from: https:// www.foodsafety.com.au/blog/the-seven-principles-of-haccp. Accessed July 25, 2020.

[23] Smith R, Bertilone C, Robertson AG. Fulminant liver failure and transplantation after use of dietary supplements. Med J Aust 2016;204(1):30-32. doi:10.5694/mja15.00816.

[24] Supporting document 3. Eligible Food Criteria - Proposal P1024, Revision of the Regulation of Nutritive Substances \& Novel Foods 2012 Available from: https://www.foodstandards.gov.au/code/proposals/ Documents/P1024\%20Nuts\%20and\%20novels\%20SD3\%20Eligible\%20Food\%20Criteria.pdf. Accessed July 25, 2020.

[25] Yoto A, Motoki M, Murao S, Yokogoshi H. Effects of L-theanine or caffeine intake on changes in blood pressure under physical and psychological stresses. J Physiol Anthropol 2012;31(1):28. doi:10.1186/1880-6805-31-28.

[26] Young HA, Benton D. Heart-rate variability: a biomarker to study the influence of nutrition on physiological and psychological health? Behav Pharmacol 2018;29(2 and 3-Spec Issue):140-151. doi:10.1097/ FBP. 0000000000000383 .

[27] Rahman F, Pechnik S, Gross D, Sewell L, Goldstein DS. Low frequency power of heart rate variability reflects baroreflex function, not cardiac sympathetic innervation. Clin Auton Res 2011;21(3):133-141. doi:10.1007/s10286-010-0098-y.

[28] Schall JD. Production, control, and visual guidance of saccadic eye movements. ISRN Neurol 2013;2013:752384. doi:10.1155/2013/752384.

[29] Liversedge SP, Findlay JM. Saccadic eye movements and cognition. Trends Cogn Sci 2000;4(1):6-14. doi:10.1016/s1364-6613(99)014187.

[30] McKune AJ, Bach CW, Semple SJ, Dyer BJ. Salivary cortisol and alphaamylase responses to repeated bouts of downhill running. Am J Hum Biol 2014;26(6):850-855. doi:10.1002/ajhb.22605.

[31] Oakley RH, Cidlowski JA. The biology of the glucocorticoid receptor: 
Explor Res Hypothesis Med

new signaling mechanisms in health and disease. J Allergy Clin Immunol 2013;132(5):1033-1044. doi:10.1016/j.jaci.2013.09.007.

[32] Russell G, Lightman S. The Human Stress Response. Nat Rev Endocrinol 2019;15(9):525-534. doi:10.1038/s41574-019-0228-0.

[33] Giles GE, Mahoney CR, Brunye TT, Taylor HA, Kanarek RB. Caffeine and theanine exert opposite effects on attention under emotional arousal. Can J Physiol Pharmacol 2017;95(1):93-100. doi:10.1139/ cjpp-2016-0498.

[34] Wing LM, Reid CM, Ryan P, Beilin LJ, Brown MA, Jennings GL, et al. Second Australian National Blood Pressure Study (ANBP2). Australian Comparative Outcome Trial of ACE inhibitor- and diuretic-based treatment of hypertension in the elderly. Management Committee on be-
Williams J. et al: Physiological responses of L-theanine: a protocol half of the High Blood Pressure Research Council of Australia. Clin Exp Hypertens 1997;19(5-6):779-791. doi:10.3109/10641969709083186.

[35] Sookan T, McKune AJ. Heart rate variability in physically active in dividuals: reliability and gender characteristics. Cardiovasc J Afr 2012;23(2):67-72.

[36] Elliott DB, Sanderson K, Conkey A. The reliability of the Pelli-Robson contrast sensitivity chart. Ophthalmic Physiol Opt 1990;10(1):21-4. doi:10.1111/j.1475-1313.1990.tb01100.x.

[37] D'Cunha NM, McKune AJ, Isbel S, Kellett J, Georgousopoulou EN, Naumovski N. Psychophysiological Responses in People Living with Dementia after an Art Gallery Intervention: An Exploratory Study. J Alzheimer's Dis 2019;72:549-562. doi:10.3233/JAD-190784. 